\title{
Risk Assessment of the Bureau Storm Disaster by the Application of Extension Theory in Sichuan
}

\author{
Tiangui Xiao ${ }^{1}$, Fengrong Jing ${ }^{1}$, Yawen $\mathrm{Zhu}^{1}$,Yujie Fang ${ }^{2}$,Wan $\mathrm{Li}^{1}$ \\ ${ }^{1}$ Chengdu University of Information Technology Atmospheric Sciences Academy, Chengdu 610225, China \\ ${ }^{2}$ Bureau of Meteorology in Pixian, Pixian 611700, China
}

\section{可拓理论在四川局地暴雨灾害风险评估中的应用研究}

\author{
肖天贵 ${ }^{1}$, 敬枫蓉 ${ }^{1}$, 朱雅文 ${ }^{1}$, 房玉洁 ${ }^{2}$, 李婉 ${ }^{1}$ \\ 1 成都信息工程大学大气科学学院, 成都 610225 , 中国 \\ 2 成都市䄮县气象局，䄮县 $611700 ，$ 中国
}

\begin{abstract}
Based on 1966-2013 daily precipitation data of 141 sites in Sichuan, GIS elevation and social statistics in Sichuan, extension theory and analytic hierarchy process, return period, correlation function and other methods to build the risk evaluation model of the bureau storm of local heavy rain in Sichuan and application analysis. Extension can create process models in selected hazard factors, disaster environment hazard bearing body, disaster prevention and mitigation capability assessment as a factor, the total amount of heavy rain, the average amount of heavy rainfall, the maximum daily amount of rain, topography, slope, population density, places are GDP, the proportion of arable land, per capita GDP, highway mileage rating parameters studied risk assessment criteria as a secondary factor assessment, calculation of AHP rights assessment factor weight coefficient, comprehensive study of the correlation function rainstorm disaster risk rating, indicating the occurrence of heavy rain in Sichuan disaster is the result of various factors interaction. Application results show that the extension theory is an effective risk assessment methodology.

Keywords: Rainstorm disaster; risk assessment; extension theory; Sichuan
\end{abstract}

摘要

利用 1966 年-2013 年四川省 141 站点的逐日降水资 料、GIS 高程及四川省社会统计资料, 基于可拓理论 及层次分析、重现期、关联函数等方法构建了四川省 局地暴雨灾害风险等级评估模型, 并进行了应用分 析。可拓模型的建立过程中选取了致灾因子、孕灾环 境、承灾体、防灾减灾能力作为一级评估因子, 暴雨 过程总量、暴雨过程平均量、最大日暴雨量、地形、 坡度、人口密度、地均 GDP、耕地比例、人均 GDP、 公路里程作为二级评估因子研究了风险评估参数分 级标准, 层次分析法计算评估因子的权重系数, 综合 关联函数研究了暴雨灾害风险等级, 表明四川暴雨灾 害的发生是多种因子共同作用的结果。应用结果表明 可拓理论是一种有效的风险评估方法。

关键词: 暴雨灾害; 风险评估; 可拓理论; 四川地区

\section{1. 引言}

暴雨是四川地区最为显著的气象灾害, 其暴雨灾 害风险评估不仅是气象业务工作的需要, 也在理论上 需要奠定坚实的基础。有关灾害风险评估的问题, 近 年来在理论和实践中都取得了很大的进展。如刘伟东 等 ${ }^{[1]}$ 合理地开始将灰色关联度的方法运用于北京地 区大风以及暴雨灾害风险等级的受损评估; 吴哲红等 ${ }^{[2]}$ 也通过灰色关联度的方法成功划分安顺市 5 个暴雨 灾情等级, 建立评估以及预评估系统, 并成功投入使 


\section{Risk Analysis and Crisis Response in Big Data Era (RAC-16)}

用; 蒋新宇等 ${ }^{[3]}$ 综合运用 GIS 技术及相关方法绘制出 松花江的干流流域关于暴雨洪涝灾害的风险区划图; 孟翠丽等 ${ }^{[4]}$ 综合运用主成分分析法 (EOF)、层次分 析法（AHP）以及 ArcGIS 技术建立了广西在农业方 面的暴雨灾害风险等级的实时评估模型; 刘荆等 ${ }^{[5]}$ 通过相关分析法以及加权求和法对淮河流域暴雨灾 害进行风险评估。Wei Zhang ${ }^{[6]}$ 等通过精确的城市防 洪风险评估模型对城市防洪管理做出了巨大贡献。 Sijian Zhao ${ }^{[7]}$ 等通过 IDW 方法, 对洪涝灾害引起的灾 害进行风险评估。杨志双等 ${ }^{[8]}$ 运用可拓理论等方法, 对西南典型山区泥石流灾害进行风险评估; 白利平等 [9]将可拓理论运用于建立北京市泥石流预警预报模 型; 孙伟等 ${ }^{[10]}$ 利用可拓理论建立了划分为五个等级的 台风灾害评估模型。但上述工作中, 尤其是西南地区, 还没有专门针对气象暴雨灾害的可拓理论风险评估 的应用工作, 因此本文利用可拓理论方法, 选取有效 评估因子建立四川局地暴雨灾害风险评估模型开展 暴雨灾害的风险评估工作, 对气象灾害评估业务的开 展有重要的实践意义。

\section{2. 数据资料和研究方法}

本文降水资料来自四川省 1966-2013 年的 141 个气象 观测站逐日降水资料，包括川西高原 31 个高原气象 观测站及 110 个非高原气象观测站。人口、耕地面积、 行政面积、GDP、公路里程等社会经济资料取自中国 统计出版社出版、四川省统计局编著的四川省统计年 鉴; 文中还使用了地理信息系统中地形高程数据、坡 度数据等信息。文中基于蔡文教授提出的可拓理论研 究了四川局地暴雨灾害风险评估模型的建立和应用。 具体的步骤如下:

（1）评估因子经典域和节域的确定

假设暴雨灾害风险等级的评价元素有 $n$ 个, 即为 $x_{1}, x_{2}, \ldots, x_{n}$, 暴雨灾害风险等 $m$ 个, 因此可得出第 $\mathrm{j}$ 个等级的物元模型中评估因子的经典域为:

$$
\begin{gathered}
R_{j}=\left(N_{j}, x, V_{j}\right)=\left[\begin{array}{ccc}
N_{j} & x_{1} & V_{1 j} \\
0 & x_{2} & V_{2 j} \\
\vdots & \vdots & \vdots \\
0 & x_{n} & V_{n j}
\end{array}\right]=\left[\begin{array}{ccc}
N_{j} & x_{1} & V_{1 j} \\
0 & x_{2} & V_{2 j} \\
\vdots & \vdots & \vdots \\
0 & x_{n} & V_{n j}
\end{array}\right]= \\
{\left[\begin{array}{ccc}
N_{j} & x_{1} & \left(a_{1 j}, b_{1 j}\right) \\
0 & x_{2} & \left(a_{2 j}, b_{2 j}\right) \\
\vdots & \vdots & \vdots \\
0 & x_{n} & \left(a_{n j}, b_{n j}\right)
\end{array}\right],(1)}
\end{gathered}
$$

式子中: $N_{i}$ 为暴雨灾害风险度评估等级的第个等 级; $x_{i}$ 为暴雨灾害风险评估等级 $N_{j}$ 的第 $i$ 个评价因子; $V_{j i}$ 为 $N_{j}$ 关于 $x_{i}$ 所规定的量值范围。
节域模型是所有暴雨灾害风险等级对于各评估 因子的取值范围, 即:

$$
R_{p}=\left(P, x, V_{p}\right)=\left[\begin{array}{ccc}
P & x_{1} & V_{1 P} \\
0 & x_{2} & V_{2 P} \\
\vdots & \vdots & \vdots \\
0 & x_{n} & V_{n P}
\end{array}\right], \quad(2)
$$

(2) 待评估的暴雨灾害物元:

$$
R=\left[\begin{array}{ccc}
p & x_{1} & v_{1} \\
0 & x_{2} & v_{2} \\
\vdots & \vdots & \vdots \\
0 & x_{n} & v_{n}
\end{array}\right],(3)
$$

(3) 待评估暴雨灾害风险评估各等级的综合关 联程度计算及风险等级的确定:

为了确定待评估暴雨灾害的等级, 比较待评估暴 雨灾害和所建立物元模型, 计算各评价元素与这 5 个等级的关联度 $K_{i}$, 再根据各评价元素的权重系数, 计算待评价暴雨灾害各个级别的综合关联程度并比 较。设待评估暴雨灾害评估因子为 $v_{i}, p$ 与 $R_{j}$ 关于评 估因子 $X$ 的距为 $\rho\left(v_{i}, V_{i j}\right), p$ 与 $R_{p}$ 关于评估因子 $X$ 的距为 $\rho\left(v_{i}, V P_{j}\right)$, 若 $v_{i}$ 属于 $V_{i j}$, 则待评估的暴雨 灾害与各暴雨灾害风险评估等级的关联函数为式 (4), 若 $\mathrm{v}_{i}$ 不属于 $V_{i j}$, 则为式 (5)。

$$
\begin{array}{r}
K_{j}\left(v_{i}\right)=-\frac{\rho\left(v_{i}, v_{i j}\right)}{\left|V_{i j}\right|}, \\
K_{j}\left(v_{i}\right)=\frac{\rho\left(v_{i}, V_{i j}\right)}{\rho\left(v_{i}, V_{P_{j}}\right)-\rho\left(v_{i}, V_{i j}\right)},
\end{array}
$$

式中 $\rho\left(v_{i}, V_{i j}\right)=\left|v_{i}-\frac{a_{i j}+b_{i j}}{2}\right|-\frac{b_{i j}-a_{i j}}{2}$; $\rho\left(v_{i}, V_{P_{j}}\right)=\left|v_{i}-\frac{a_{P_{j}}+b_{P_{j}}}{2}\right|-\frac{b_{P_{j}}-a_{P_{j}}}{2} ;\left|v_{i j}\right|=\left(b_{i j}-a_{i j}\right) 。$

设 $\lambda_{i}$ 为评价因子 $X$ 的权重系数, 则待评估暴雨 灾害关于暴雨灾害等级 $j$ 的综合关联隶属度为式 (6)：

$$
K_{j}(p)=\sum_{i=1}^{n} \lambda_{i} K_{i}\left(v_{i}\right), \quad(6)
$$

如果 $K_{j 0}(p)=\max _{j=(1,2, \ldots, m)} K_{j}(p)$, 则待评估暴雨 灾害 $p$ 属于 $j 0$ 等级。

\section{3. 暴雨灾害的可拓性特征}

按可拓的观点, 物元的可拓性即为事物变化的可 能性, 包括物元的可扩性、发散性、共轭性和相关性 [11-13], 因此, 研究暴雨灾害风险评估问题时, 需要从 暴雨灾害出发向外、向内、平行、变换以及组合的思 维方法分析暴雨灾害拓展的各类可能性, 分析暴雨灾 
Risk Analysis and Crisis Response in Big Data Era (RAC-16)

害的特征以及暴雨灾害的可影响因素物元的可拓性, 即暴雨灾害的可扩性、发散性、共轭性和相关性。在 考虑量变的同时也考虑质变的问题, 建立一个形式化 的模型去完成量变到质变的具体转化过程 [14-15]。

暴雨灾害的可扩性。暴雨灾害的产生由致灾、孕 灾、承灾体与防灾减灾能力四个方面综合作用, 而致 灾因素与暴雨本身等级、频繁程度等息息相关; 孕灾 环境则与地形和坡度等紧密联系着; 承灾体则受地方 社会经济等的影响; 防灾减灾体系则与人均 GDP、 交通系统相联系。可见, 与暴雨灾害有直接联系的评 估因子都是可以层层扩展的。

暴雨灾害的发散性。物元的发散性是指一物元拥 有多种不同特征、一种特征又会被多个物元所具备。 在不同的地方可能会发生相同的暴雨灾害, 表现出一 特征多物元的特点; 相同的暴雨灾害也会造成有差异 的经济损失, 产生不同的等级差异, 表现出一物多征 的特点。因此暴雨灾害具有发散性。

暴雨灾害的共轭性。对暴雨灾害风险评估中的地 形、坡度等评价元素反映的是自然属性, 因此是绝对 的; 而致灾因素、能承受灾害的程度以及防灾和减灾 能力等都是反映的该地区的社会属性, 在不同地区差 异较大。相同的暴雨在不同的站点时, 产生的风险等 级不同, 因此又是相对的。这就是暴雨灾害的潜显共 轭性。

暴雨灾害的相关性。不同事物关于某一种特征的 值域之间，同一种或同类型的事物关于某一些特征的 值域之间, 如果存在一定程度的相互依赖, 则相关。 而暴雨灾害的风险等级与暴雨强度、㝋灾的环境、能 承受灾害的程度以及防灾减灾能力均密切相关, 因此 暴雨灾害又是具有相关性的。

可见, 基于暴雨灾害的可扩性、发散性、共轭性 以及相关性的特征, 可拓理论适用于暴雨灾害风险评 估模型的建立。

\section{4. 可拓局地暴雨灾害风险评估模型的建立}

根据可拓理论和暴雨灾害的特征, 暴雨灾害可拓 评估模型建立的基本思路是: 根据四川历史暴雨降水 数据以及高程、年鉴统计资料等确定各评估参数的经 典域与节域; 确定各评估因子权重系数; 将评估因子 的具体信息数据带入各数据类中, 计算关联度及综合 关联度; 比较各个风险等级的综合关联程度值, 获得 待评估模型综合关联程度最大的等级, 即为其风险等 级。

本文考虑四川局域即单站暴雨灾害的评估, 评估 因子选取暴雨过程总量、平均量、最大日暴雨量、地
形、坡度、地均 GDP、人口密度、耕地比重、人均 GDP、公路里程等十个指标。首先确定暴雨灾害评估 等级的划分, 然后从 1966-2013 年四川省 141 单站降 水资料挑选出符合单站暴雨的数据, 根据历史暴雨统 计资料通过正态分布函数确定致灾因子中单站单次 暴雨过程总量、平均量、最大日暴雨量不同重现期一 2 年一遇、 10 年一遇、 20 年一遇、 50 年一遇的等级 划分成 5 个等级的值。同时对地形、坡度、地均 GDP、 人口密度、耕地比重、人均 GDP、公路里程确定经 典域等级划分, 利用层次分析法获得十个评估影响因 子的权重, 然后通过关联函数可得待评价暴雨灾害的 风险等级。

\section{1. 灾害评估等级的划分}

暴雨灾害造成的危害及损害不只与暴雨本身的雨量 分布有关, 而是一个与社会所处状况相关值, 与暴雨 灾害发生地区的经济发展、人口密集等社会属性密不 可分。根据气象部门规定, 按照人员伤亡、经济损失 的多少, 将暴雨灾害划分成 5 级。但由于四川地区有 着东部西部地区不同的情况可作适当的调整, 因此, 将致灾因子、承灾体、孕灾环境及防灾减灾力作为暴 雨灾害风险评估作为评估标准, 分为 5 个等级。等级 越大, 风险越高。

表 1 气象灾害评估标准

\begin{tabular}{|c|c|c|c|c|c|}
\hline & 特大型 & 大型 & 中型 & 小型 & 较小型 \\
\hline $\begin{array}{l}\text { 死亡 } \\
\text { 人/人 }\end{array}$ & {$[100,+\infty)$} & {$[30,100)$} & {$[3,30)$} & {$[1,3)$} & \\
\hline $\begin{array}{l}\text { 伤亡 } \\
\text { 人/人 }\end{array}$ & {$[300,+\infty)$} & {$[100,300)$} & {$[30,100)$} & {$[10,30)$} & {$[1,10)$} \\
\hline $\begin{array}{c}\text { 直接 } \\
\text { 损失/ } \\
\text { 万元 }\end{array}$ & {$[105,+\infty)$} & {$[104,105)$} & {$[103,104)$} & {$[102,103)$} & {$[101,102)$} \\
\hline
\end{tabular}

将单站暴雨过程总量、平均量、最大日暴雨量作为致 灾因子, 反映暴雨强度, 将重现期当作致灾因子评估 其等级的标准。但在四川地区, 由于川西高原与四川 盆地的地域差异, 高原站与非高原站单站暴雨过程采 取了不同的定义, 高原站日降雨量大于或等于 25 毫 米及以上，持续时间大于等于 1 天称为暴雨过程; 在 非高原站日降雨量大于等于 50 毫米及以上, 持续时 间大于等于 1 天称为暴雨过程。

选取 1966-2013 年四川 141 个站点的暴雨资料, 统计其频数分布, 分别可得 141 个单站暴雨过程总 量、平均暴雨量、最大日暴雨量的频数分布图, 分布 图均呈典型的偏态分布。通过分位数的计算, 使得 3 
Risk Analysis and Crisis Response in Big Data Era (RAC-16)

个评估指标的数据分布转化为正态分布, 并通过 K-S (Kolmogorov-Smirnov) 非参数检验法进行了显著性 检验, 均通过 0.05 信度下的对数正态分布假设, 进 一步获得了使用对数正态分布函数计算重现期的分 布特征。

根据重现期的概念, 将 3 个评估指标的五个等级 分别定为小于 2 年一遇、介于 2 年一遇到 10 年一遇 之间、介于 10 年一遇到 20 年一遇之间、介于 20 年 一遇到 50 年一遇及介于 50 年一遇到 100 年一遇之间 的值, 然后确定 3 个指标因子发生这五个重现期等级 暴雨所对应的概率值得到它们所对应的分位数作为 五个重现期对应理论值, 最终由 5 个重现期划分而成 的等级值作为四川省暴雨灾害风险评估致灾因子中 的评估标准, 成为评估模型中的一部分。

\section{3. 评估因子经典域的划分}

由致灾因子中单站总暴雨量、平均暴雨量、最大 日暴雨量, 结合承灾体的地形、坡度评估指标, 所属 孕灾环境的人口密度、地均 GDP、耕地比重, 以及 体现防灾减灾力的人均 GDP、公路里程等 10 个评估 指标, 形成雨灾害风险评估指标 5 个等级经典域, 如 表 2 所示。

\begin{tabular}{|c|c|c|c|c|c|}
\hline 因素 & 一级 & 二级 & 三级 & 四级 & 五级 \\
\hline $\begin{array}{c}\text { 总暴雨量 } \\
\text { (等级) }\end{array}$ & 1 & 2 & 3 & 4 & 5 \\
\hline $\begin{array}{l}\text { 平均暴雨 } \\
\text { 量(等级) }\end{array}$ & 1 & 2 & 3 & 4 & 5 \\
\hline $\begin{array}{c}\text { 最大日暴 } \\
\text { 雨量(等级) }\end{array}$ & 1 & 2 & 3 & 4 & 5 \\
\hline $\begin{array}{l}\text { 人口密度 } \\
\text { (人/km2) }\end{array}$ & $0 \sim 265$ & $265 \sim 415$ & $415 \sim 581$ & $581 \sim 738$ & $738 \sim 1499$ \\
\hline $\begin{array}{l}\text { 地均 GDP } \\
\text { (万元 } / \mathrm{km} 2 \text { ) }\end{array}$ & $\begin{array}{l}2118 \sim \\
14602\end{array}$ & $\begin{array}{l}1204 \sim \\
2118\end{array}$ & $\begin{array}{l}826 \sim \\
1204\end{array}$ & $364 \sim 826$ & $0 \sim 364$ \\
\hline $\begin{array}{c}\text { 耕地比例 } \\
(\%)\end{array}$ & $0 \sim 0.118$ & $\begin{array}{l}0.118 \sim \\
0.192\end{array}$ & $\begin{array}{l}0.192 \sim \\
0.255\end{array}$ & $\begin{array}{c}0.255 \sim \\
0.329\end{array}$ & $\begin{array}{c}0.329 \sim \\
0.526\end{array}$ \\
\hline 坡度(度) & $\begin{array}{l}16.80 \sim \\
48.74\end{array}$ & $\begin{array}{l}7.25 \sim \\
16.80\end{array}$ & $\begin{array}{c}2.81 \sim \\
7.25\end{array}$ & $\begin{array}{c}0.93 \sim \\
2.81\end{array}$ & $0 \sim 0.93$ \\
\hline 地形(米) & $1655 \sim$ & $593 \sim$ & $488 \sim 593$ & $396 \sim 488$ & $0 \sim 396$ \\
\hline 人均 & 3782 & 1655 & $22315 \sim$ & $16420 \sim$ & $0 \sim 16420$ \\
\hline GDP(元) & $32877 \sim$ & $26774 \sim$ & 26774 & 22315 & $0 \sim 920$ \\
\hline $\begin{array}{c}\text { 公路里程 } \\
(\mathrm{km})\end{array}$ & $\begin{array}{c}106106 \\
3333 \sim \\
19449\end{array}$ & $\begin{array}{c}32877 \\
2387.85 \\
\sim 3333\end{array}$ & $\begin{array}{c}1561 \sim \\
2387.85\end{array}$ & $\begin{array}{l}920 \sim \\
1561\end{array}$ & \\
\hline
\end{tabular}

4.4. 权重系数的建立

利用层次分析法, 将致灾因子、承灾体、孕灾环境以
及防灾减灾能力作为第一层评估指标, 将暴雨总量、 暴雨平均量、最大日暴雨量、地形、坡度、人口密度、 地均 GDP、耕地比例、人均 GDP、公路里程作为第 二层评估因子, 通过建立层次结构模型, 构造判断矩 阵, 计算权重向量并作一致性检验, 最终获得了表 3 的两个层次级别元素的权重系数, 一级指标中, 致灾 因子对灾害风险的影响最大, 而人为的防灾减灾能力 的影响所占权重最小。二级因子中, 暴雨总量、暴雨 平均量、最大日暴雨量、地形、坡度、人口密度、地 均 GDP、耕地比例、人均 GDP、公路里程 10 个因子 权重系数为: $0.138636 、 0.076294 、 0.251918 、 0.0462$ 、 $0.2313 、 0.0916 、 0.0458 、 0.0229 、 0.0238 、 0.0715$ 。 其中致灾因子所占一级评估因子的权重最大, 影响力 最深, 依次减少的一级指标是孕灾环境、承灾体、防 灾减灾力。在 10 个二级评估指标中, 最大日暴雨量 占暴雨灾害风险评估最大权重, 暴雨总量其次。

表 3 暴雨灾害风险评估两级因子的权重系数

\begin{tabular}{cccc}
\hline 一级指标 & 权重系数 & 二级指标 & 权重系数 \\
\hline 致灾因子 & 0.4668 & 暴雨总量 & 0.297 \\
& & 暴雨平均量 & 0.163 \\
最大日暴雨量 & 0.540 \\
承灾伓境 & \multirow{2}{*}{0.2776} & 地形 & 0.1667 \\
& & 坡度 & 0.8333 \\
& \multirow{2}{*}{0.1603} & 人口密度 & 0.5714 \\
防灾减灾能力 & \multirow{2}{*}{0.0953} & 地均 GDP & 0.2857 \\
& & 耕地比例 & 0.1429 \\
& & 人均 GDP & 0.25 \\
& & 公路里程 & 0.75 \\
\hline
\end{tabular}

\section{5. 可拓暴雨物元模型的建立}

待评估暴雨灾害有 5 个风险等级的可能,其影响 因子有暴雨总量 (rsum)、暴雨平均量 (rave) 、最大 日暴雨量 $(\mathrm{rmax})$ 、地形 $(\mathrm{dx})$ 、坡度 $(\mathrm{pd})$ 、人口密度 (rk)、地均 GDP (djg)、耕地比例 ( gd )、人均 GDP (rjg)、 公路里程 $(\mathrm{gl})$, 综合这 10 个评估因子的值, 即体现 了暴雨灾害的特征, 从而由这 10 个指标的值构成了 暴雨灾害的物元模型, 量化对应的 10 个评估因子, 即构成了待评估暴雨灾害的物元特征值, 构造了四川 省暴雨灾害风险评估模型（表 4)。

\section{5. 可拓局地暴雨灾害模型的应用}

根据四川省暴雨灾害风险评估模型,基于可 拓理论原理，从致灾因子、孕灾环境、承灾体、防灾 减灾能力四个方面对广元旺苍县站 2014 年 9 月 10 日至 11 日发生的暴雨进行风险等级的评估。旺苍站 10 日降水 $121.9 \mathrm{~mm}, 11$ 日降水 $65.4 \mathrm{~mm}$, 因此, 暴雨 过程总量为 $187.3 \mathrm{~mm}$, 暴雨过程平均量为 $93.7 \mathrm{~mm}$, 
Risk Analysis and Crisis Response in Big Data Era (RAC-16)

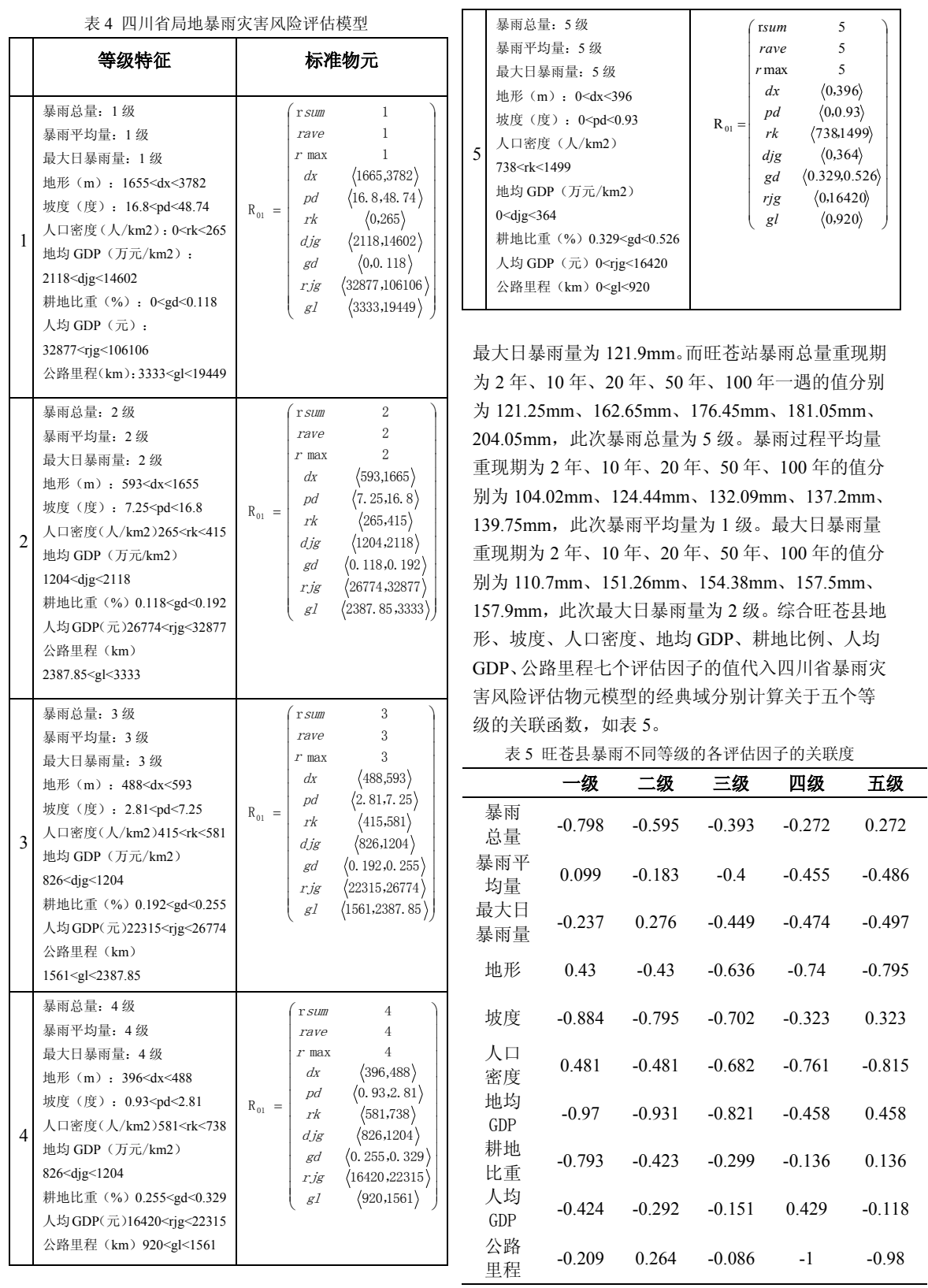


Risk Analysis and Crisis Response in Big Data Era (RAC-16)

利用上表以及所得暴雨总量、暴雨平均量、最大 日暴雨量、地形、坡度、人口密度、地均 GDP、耕 地比例、人均 GDP、公路里程 10 个因子的权重系数 $0.139 、 0.076 、 0.252 、 0.046 、 0.231 、 0.092 、 0.046$ 、 $0.023 、 0.024 、 0.072$ 可得待评估暴雨灾害关于 5 个暴 雨风险等级的关联程度。

\begin{tabular}{|c|c|c|c|c|c|}
\hline & 一级 & 二级 & 三级 & 四级 & 五级 \\
\hline $\begin{array}{c}\text { 关联函 } \\
\text { 数值 }\end{array}$ & -0.439 & -0.337 & -0.518 & -0.465 & -0.162 \\
\hline
\end{tabular}

因此, 根据可拓理论中关联函数的定义, 此次旺 苍县评估的暴雨灾害风险等级为第五级暴雨。而根据 历史灾情资料, 此次旺苍县直接经济损失为 3.98 亿 元, 死亡 2 人, 受灾人数达 183500 人, 被困 2159 人, 农田受灾面积 581.8 亩。因此这次暴雨引起了旺 苍县多处山洪暴发、河水猛涨，甚至大部分的乡间公 路中断、多数村民房屋被摧毁, 电力设施被损坏、农 田被淹、农作物被破坏, 一些乡镇甚至进水、交通、 通讯也终端, 根据表 1 实况评估为 5 级。因此, 通过 基于可拓理论的暴雨灾害风险评估结果是有效的。

\section{6. 结论与讨论}

基于可拓模型的四川暴雨灾害风险评估模型的 建立是非常有意义的工作, 对于气象业务有重要的促 进作用。文中选取致灾因子、孕灾环境、承灾体、防 灾减灾能力作为一级评估因子, 并将单站暴雨过程总 量、暴雨过程平均量、最大日暴雨量、地形、坡度、 人口密度、地均 GDP、耕地比例、人均 GDP、公路 里程作为二级评估因子, 并通过区域暴雨特征分析提 出的高原站和非高原站的暴雨灾害分级、通过对数正 态分布函数获得重现期作为致灾因子等级评估标准 等思路, 对业务应用都是有实践意义的。但由于暴雨 灾害影响的复杂性以及致灾因子资料收集的困难, 风 险评估模型建立的恰当还需进一步研究。目前, 我们 已经尚试对可拓暴雨灾害模型进行了应用研究, 并在 暴雨灾害评估系统中初步应用。

\section{Acknowledgements}

This study was supported by Southwest Regional Meteorological Center of China Meteorological Administration major research projects (Southwest Region014-5)、National Natural Science Foundation of China Fund Project (91337215),and the National Key Basic Research Program (2013CB733206), Sichuan Province, applied basic research projects (2013JY0063)。

\section{致谢}

本研究得到了中国气象局西南区域气象中心重 大科研项目（西南区域 014-5）、国家自然科学基金 重点基金项目（91337215）、国家重点基础研究发展 计划（2013CB733206）、四川省应用基础研究计划 项目（2013JY0063）的资助。

\section{参考文献}

[1] 刘伟东，扈海波，程从兰，等. 灰色关联度方 法在大风和暴雨灾害损失评估中的应用. 气象 科技, 2007, 04: 563-566.

[2] 吴哲红, 陈贞宏, 叶帮, 等. 基于灰色关联度 的暴雨事件灾害评估和预评估方法. 贵州气象, 2011, 06:6-9.

[3] 蒋新宇, 范久波, 张继权, 等. 基于 GIS 的松 花江干流暴雨洪涝灾害风险评估. 灾害学, 2009, 03: 51-56.

[4] 孟翠丽, 匡昭敏, 李莉，等. 基于 GIS 的广西 暴雨灾害风险实时评估技术研究. 中国农学通 报, 2013, 26: 184-189.

[5] 刘荆, 蒋卫国, 杜培军, 等. 基于相关分析的 淮河流域暴雨灾害风险评估. 中国矿业大学学 报, 2009, 05: 735-740.

[6] W. Zhang, Y.J. Hu, J.H. Wang, C.Y. Zhang. Research on Urban waterlogging disaster risk assessment based on ARCGIS and MIKE FLOOD - A case study on Shijiazhuang. Journal of Risk Analysis and Crisis Response,2015,5-4:226-233.

[7] S.J. Zhao, Q. Zhang. Risk assessment of crops induced by flood in the three northeastern provinces of China on small space-and-time scales. Journal of Risk Analysis and Crisis Response, 2012,2-3:201-208

[8] 杨志双, 韩玉龙, 张浩然. 基于可拓理论的西 南典型山区泥石流的危险性分级. 河南理工大 学学报(自然科学版), 2013, 01: 35-39.

[9] 白利平，王业耀，戴斌，等. 基于可拓理论的 泥石流灾害预警预报系统开发: 以北京市为例. 现代地质, 2009, 01: 157-163.

[10] 孙伟，高峰，刘少军，等. 海南岛台风灾害损 失的可拓评估方法及应用. 热带作物学报, 2010 , 02: 319-324.

[11] 蔡文. 可拓论及其应用. 科学通报, 2004, 07 673-682.

[12] 李琴, 王国会, 陈清华. 可拓分类方法及其在 流动单元分类中的应用. 地球物理学进展, 2007, 06: 1975-1979.

[13] 刘少军. 基于多信息源的台风灾害实时评估系 统研究. 成都理工大学, 2011 .

[14] 孙绍骋. 灾害评估研究内容与方法探讨. 地理科 学进展, 2001, 02: 122-130.

[15] 陈艳秋,袁子鹏,盛永,等. 辽宁暴雨事件影响的预 评估和灾后速评估. 气象科学, 2007, 06: 626-632. 